\section{BMJ Open Respiratory Research}

\title{
Developing an intrasalivary gland botox service for patients receiving long-term non-invasive ventilation at home: a single-centre experience
}

\author{
Jessica Harbottle, ${ }^{1}$ Hannah Carlin, ${ }^{2}$ Thomas Payne-Doris, ${ }^{3}$ Hilary M I Tedd, ${ }^{2}$ \\ Anthony de Soyza, ${ }^{4}$ Ben Messer ${ }^{3}$
}

To cite: Harbottle J, Carlin $\mathrm{H}$ Payne-Doris T, et al. Developing an intrasalivary gland botox service for patients receiving long-term noninvasive ventilation at home: a single-centre experience. BMJ Open Resp Res 2022;9:e001188. doi:10.1136/ bmjresp-2021-001188

Received 25 December 2021 Accepted 31 January 2022
Check for updates

(c) Author(s) (or their employer(s)) 2022. Re-use permitted under CC BY-NC. No commercial re-use. See rights and permissions. Published by BMJ.

${ }^{1}$ School of Medical Education, Newcastle University, Newcastle upon Tyne, UK ${ }^{2}$ Respiratory Medicine, Newcastle upon Tyne Hospitals NHS Trust, Newcastle upon Tyne, UK ${ }^{3}$ North East Assisted Ventilation Service, Newcastle upon Tyne NHS Hospitals Foundation Trust, Newcastle upon Tyne, UK

${ }^{4}$ Population Health Science Institutes, Newcastle University, Newcastle upon Tyne, UK

Correspondence to Dr Ben Messer; ben.messer@nhs.net

\section{ABSTRACT}

Introduction Sialorrhoea is a debilitating symptom in neurological disease and there is a growing literature for the use of intrasalivary gland Botulinum Toxin (botox) injections in its management. However, provision of intrasalivary gland botox remains inconsistent and sialorrhoea is often poorly controlled in motor neuron disease (MND).

Sialorrhoea in association with bulbar dysfunction can cause intolerance of non-invasive ventilation (NIV) and respiratory infection, so its treatment is critical within a home ventilation service (HVS).

This treatment can also be used to enable tracheostomy cuff deflation to facilitate weaning from ventilation. We report on the outcomes of intrasalivary gland botox in our HVS.

Methods In 2015, we set up an intrasalivary gland botox service for patients under our HVS. Under ultrasound guidance, we injected submandibular gland (SMG), parotid gland (PG) or both.

Results 109 intrasalivary gland botox procedures were performed in 72 patients. Diagnostic groups included MND 32

Cerebral Palsy 8 and Weaning 14. Glands injected were, SMG (6\%), PG (47\%) and both (47\%). The majority $(84 \%)$ received the Dysport preparation with mean dose 273 units. $94 \%$ were ultrasound guided. $81 \%$ of injections resulted in a positive treatment effect, with $47 \%$ patients requesting repeat injections. Complications were angioedema $(0.9 \%)$ and worsening dysphagia $(3.7 \%$ following SMG injection). Mean survival following treatment was 40 months with $53 \%$ patients still alive.

Conclusions Intrasalivary gland botox appears effective across a range of neurological conditions requiring longterm NIV with few complications. Dysphagia may be an important complication of SMG injection. A randomised controlled trial may help establish the evidence base.

\section{INTRODUCTION AND BACKGROUND}

Sialorrhoea is an excessive accumulation of saliva in the mouth and is often accompanied by drooling. Saliva is produced by submandibular, parotid and sublingual glands with minor salivary glands found in the oral cavity,
Key messages

What is already known on this topic?

- Sialorrhoea is a common and debilitating symptom in patients requiring home ventilation and is often poorly controlled. There is a growing evidence base for intrasalivary gland Botulinum Toxin (botox) in neurological conditions including Parkinson's disease and motor neuron disease but little evidence in patients receiving home ventilation.

\section{What this study adds?}

- This large cohort study of intrasalivary gland botox has shown that the ability to administer intrasalivary gland botox is an important skill within a home ventilation MDT, outcomes are good, side effects rare and almost half of patients return for repeat injections.

How this study might affect research, practice or policy?

- This study highlights an opportunity to develop the evidence base for intrasalivary gland botox in patients under the care of home ventilation services. We hope that this study will also provide guidance and inspiration for other intrasalivary gland botox services to develop for patient benefit.

for example, the labial and lingual mucosa, as well as the palate and floor of the mouth. ${ }^{1} \mathrm{Up}$ to $1.5 \mathrm{~L}$ of saliva are produced each day with the submandibular glands contributing $60 \%$ and the parotids contributing 20\%-25\%. ${ }^{1}$

Sialorrhoea is a common symptom in neurological diseases in which it arises secondary to dysphagia (reduced swallowing of saliva) although other mechanisms may contribute such as autonomic dysfunction (increased saliva production). Sialorrhoea is a debilitating and distressing symptom for patients and caregivers. $^{2-4}$

Patients who require treatment with noninvasive ventilation (NIV) or who are weaning from mechanical ventilation frequently encounter sialorrhoea. For example, in a UK 
survey of motor neuron disease (MND), the incidence of sialorrhoea was $42 \%$, and it is a recognised risk factor for aspiration pneumonia. ${ }^{56}$ Sialorrhoea, drooling and aspiration pneumonia are common in children with cerebral palsy $(\mathrm{CP})^{7}$ and sialorrhoea has also been reported in patients with genetic muscle disease. ${ }^{8}$ Finally, sialorrhoea and aspiration pneumonia are common in patients with brain injuries and are associated with worse outcomes such as delayed weaning from invasive ventilation. ${ }^{9}$ Common features across these conditions are significant healthcare costs associated with the effects of sialorrhoea in terms of morbidity, increased hospitalisation risks and prolongation of stay.

Our home ventilation service (HVS) delivers NIV and cares for more than 1000 patients and approximately a third of these have neuromuscular diseases including MND. NIV improves survival and quality of life outcomes in patients with MND and respiratory failure; however, these benefits are reduced in patients with severe bulbar dysfunction. ${ }^{10}{ }^{11}$ Sialorrhoea in MND can impact on the ability to deliver NIV. In addition, tolerance of NIV is improved in patients with lower secretion burden in the upper airway and it is, therefore, an important symptom to address. ${ }^{12}$

Almost half of patients with sialorrhoea associated with MND have uncontrolled symptoms. ${ }^{5}$ Currently, there is evidence of variation in treatment between centres. ${ }^{5} 13$ The National Institute for Health and Care Excellence (NICE) recommends glycopyrronium as the first-line treatment for sialorrhoea. ${ }^{14}$ However, antimuscarinic drugs are limited by treatment side effects $(54 \%)$ and poor efficacy $(39 \%) .{ }^{13}$ Side effects include blurred vision, constipation and confusion. These are all challenging side effects in populations suffering with sialorrhoea where cognitive impairment, sensory loss, constipation and urinary tract infections are problematic.

There is a growing literature for the use of intrasalivary gland Botulinum Toxin (botox) injections in neurological conditions including Parkinson's disease (PD), $\mathrm{CP}$ and MND. ${ }^{15}$ The Xeomin preparation of botox has recently been approved by NICE for the treatment of chronic sialorrhoea caused by neurological conditions. ${ }^{16}$ NICE management guidelines for MND, published in 2016 and updated in 2019, recommend further research into additional treatments for sialorrhoea in MND is needed. ${ }^{17}$ Notably, a Cochrane review of treatment of sialorrhoea in MND identified only one randomised controlled trial of 20 patients published in 2009. ${ }^{18}$

The Sialorrhea in Adults Xeomin Investigation (SIAXI) trial showed a significant improvement in sialorrhoea and unstimulated salivary flow rate with intrasalivary gland incobotulinumtoxinA in patients with PD and following traumatic brain injury. ${ }^{19}$ In children with $\mathrm{CP}$, a recent meta-analysis demonstrated improvements in drooling following intrasalivary gland botox injections. ${ }^{20}$

UK MND surveys have highlighted inconsistent access to intrasalivary gland botox injections as a treatment for sialorrhoea and have shown that it is not used as a first-line treatment. ${ }^{513}$ The evidence for the efficacy of intrasalivary gland botox in MND is derived from case series and Randomised Controlled Trials (RCTs) with small numbers of patients, which often coenrol patients with other neurological diseases. ${ }^{21-23}$ A systematic review undertaken in 2014 found a mean of only 12.5 patients in each study. ${ }^{24}$

In 2015, we set up an intrasalivary gland botox service, initially to benefit patients with MND and other neuromuscular conditions under the HVS. We report on the development of our service and present a retrospective review of the practice and outcomes of the first 109 intrasalivary gland botox treatments we have administered.

\section{METHODS}

Two consultants (TP-D and BM) trained in ultrasoundguided intrasalivary gland botox injections in the following ways: practical mentorship from radiology colleagues and an existing consultant within the HVS, attendance at the cadaveric lab at Newcastle University to study salivary gland anatomy and attendance in theatre to observe salivary gland surgery. Both consultants are intensivists, regularly using ultrasound in critical care settings for intravenous access.

Initially, the service was set up for patients under the HVS only, attending as day cases. The service subsequently expanded to include all patients with secretion management problems related to neurodisability in the North East of England and Cumbria. More recently, the service has been offered to regional critical care units to facilitate weaning from ventilation by enabling deflation of cuffed tracheostomies. The growth of the service is detailed in figure 1.

Patients were identified by reviewing all botox prescriptions requested by the regional HVS. The relevant patient records and home ventilation database were examined for any postprocedure assessments. A positive treatment outcome was defined as a subjective benefit reported by the patient in postprocedure consultations, or in the case of intubated critical care patients, successful tracheostomy cuff deflation, extubation or decannulation following

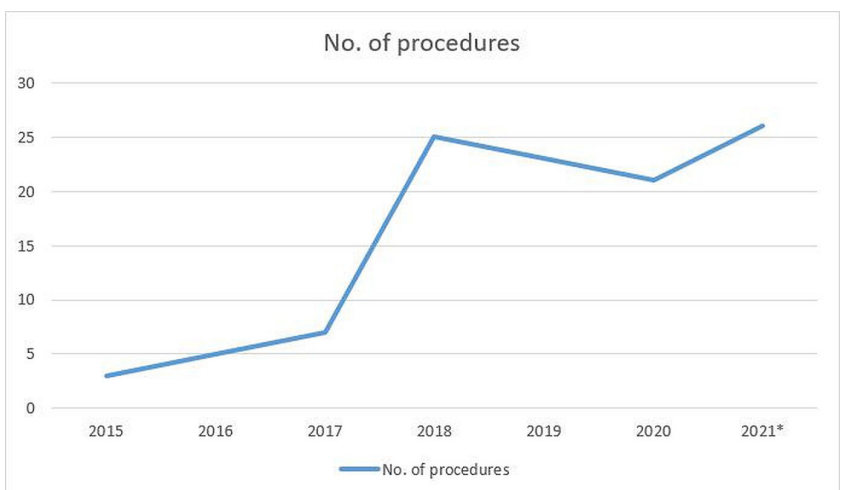

Figure 1 Number of intrasalivary gland botox injections over time. *Data from 2021 to end of July. 
Table 1 Diagnostic groups of patients undergoing intrasalivary gland botox

\begin{tabular}{lc}
\hline Diagnostic group & Number of patients \\
\hline MND & 32 \\
CP & 8 \\
Congenital muscle disease & 5 \\
Weaning & 14 \\
Other* $^{*}$ & 13 \\
\hline
\end{tabular}

${ }^{*}$ Other included a range of indications such as Intracranial haemorrhage, hypoxic brain injury and multi-system atrophy. $\mathrm{CP}$, cerebral palsy; MND, motor neuron disease.

injection. In those patients where effectiveness was not documented, but they elected to have further injections, it was assumed that their first treatment was effective. Sialometry was not undertaken due to the patient cohort being highly disabled and repeated hospital attendances, therefore being inappropriate.

Initially, both submandibular gland and parotid gland were offered as standard but following complications of submandibular gland injection (see below), parotid glands only were injected in patients with preserved swallow.

\section{RESULTS}

We have carried out 109 intrasalivary gland botox injections in 72 patients with a range of diagnoses (table 1).

\section{Pre-botox management}

Many of the patients had previously tried one or more treatments for sialorrhoea. The most commonly used treatments were glycopyrronium $(74 \%)$ and hyoscine patches $(33 \%)$ and atropine $(7 \%)$.

\section{Procedures}

Fourty-seven per cent of procedures were both submandibular and parotid gland injection, $47 \%$ were parotid gland injection alone and $6 \%$ were submandibular gland injection alone. The majority of injections used the Dysport preparation $(84 \%)$ and the remainder used the Xeomin preparation. The mean dose of Dysport used was 272.9 units with a range of $150-500$ units. The concentration used was $100 \mathrm{U} / \mathrm{mL}$. The mean dose of Xeomin used was 63.2 units with a range of 15-100 units. The concentration used was $20 \mathrm{U} / \mathrm{mL}$. Ultrasound guidance was used in $93.6 \%$ of injections.

Seventy-two per cent of treatments resulted in a positive treatment outcome, increasing to $81 \%$ when patients with no outcome data are excluded. Twenty-eight per cent of patients requested repeat injections after a mean of 3.9 months (the effect of botox typically lasts 3-6 months). However, this figure may not represent the true value of this procedure for patients. After excluding patients injected within the last 6 months (within the potential effective window of the botox), those who died within 6 months of their initial injection and those who were injected in Intensive Care Unit (ICU) as a one-off procedure to facilitate extubation, this figure increased to $47 \%$.

Serious complications were rare and included angioedema $(0.9 \%)$ and dysphagia (2.8\%-only following submandibular injection). The doses of botox were similar in cases of dysphagia to those without this complication. Other mild adverse effects included pain $(1.8 \%)$ and dry mouth $(1.8 \%)$. One patient received salivary radiotherapy due to pain on injection.

Mean survival from first injection is greater than 40 months from first injection, with 53\% patients still alive at the time of writing (figure 2).

\section{DISCUSSION}

From the literature, it is clear that many of the conditions requiring long-term ventilation are associated with a high incidence of sialorrhoea. ${ }^{5-9}$ Our HVS cares for more than 1000 patients and approximately a third of these have neuromuscular disease, with 60 patients with MND. Sialorrhoea is distressing for patients and can be associated with aspiration pneumonia and other poor clinical outcomes including difficulty with delivery of NIV. ${ }^{6} 12$ Both the published literature ${ }^{13}$ and our own experience support that incomplete symptom control and/or intolerance are common with antimuscarinic therapy in these populations. In contrast to $\mathrm{PD}$, where there are emerging data on the efficacy of intrasalivary gland botox injection, there are few data across the broader range of neuromuscular conditions requiring home ventilation.

This is to our knowledge the largest cohort study of intrasalivary gland botox and the first to report on its use in a home ventilation and weaning setting. Our data demonstrate that the use of intrasalivary gland botox in a diverse range of conditions is associated with good outcomes and few complications.

In a large proportion of procedures where outcome data were available $(81 \%)$, a positive effect was achieved. Additionally, after excluding patients who would not be expected to require a second injection (patients who died within 6 months of initial treatment, patients who were still within the expected duration of efficacy from their first injection, and patients who underwent intrasalivary gland botox to facilitate weaning); the percentage of patients opting for repeat treatment was $47 \%$.

The majority of the patients in this cohort had either a progressive neurodegenerative condition or a critical illness. Despite this, mean survival is greater than 40 months and, as shown in the Kaplan-Meier curve, 53\% of patients remain alive (figure 2). The treatment is not expected to alter life expectancy, but it can significantly improve a distressing symptom of neurological disease and potentially improve quality of life for some.

Serious adverse events were rare $(3.7 \%$ of procedures). One patient suffered from angioedema following 


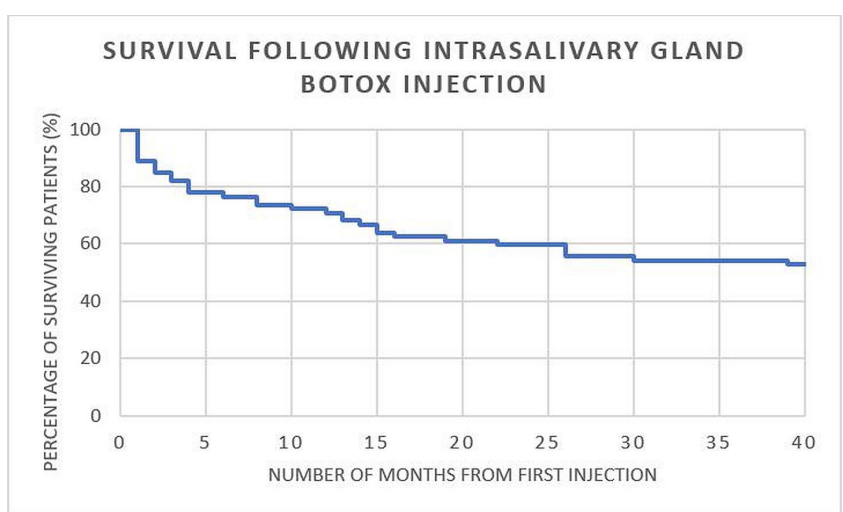

Figure 2 Survival following intrasalivary gland botox injection.

administration of botox. This was a repeat procedure after previous uncomplicated treatments. Due to the risk of idiosyncratic reactions to intrasalivary gland botox, all patients receiving the treatment are counselled on the risk and all injections are carried out in a clinical environment where emergency drugs and equipment are accessible. Three patients suffered worsening dysphagia following submandibular gland injection, two of which persisted beyond the duration of action of the administered botox. Both these patients had MND with severe bulbar dysfunction. The third patient's dysphagia improved within a few days. Following these cases, submandibular gland injections are no longer offered to any patient who still has any level of oral intake (including patients who get their primary nutrition through a gastrostomy but continue some oral intake). Understanding the causes of dysphagia after the administration of intrasalivary gland botox can be challenging. There is the potential for diffusion of botox into muscles controlling swallowing following injection, however, worsening dysphagia in MND is also an expected feature of the primary disease progression.

Given the prevalence of sialorrhoea in neuromuscular disease and, in particular, MND, ${ }^{5}$ this is a highly relevant skill to have within a HVS. The existing skills and experience of consultants within the HVS lent themselves to the development of this service particularly with regards to the use of ultrasound for identification of salivary anatomy. Ultrasound has been shown to improve the success of intrasalivary gland botox. ${ }^{25}$ Further training was also undertaken using the University cadaveric surgical skills lab and with surgical and radiology colleagues.

The majority of injections were with the Dysport preparation, but this has changed to the Xeomin preparation over time to reflect NICE guidance. ${ }^{16}$ The dose of Dysport varied between 150 and 500 units, depending on the glands injected. The dose of Xeomin followed NICE guidance when both parotid and submandibular glands were injected (100 units in total) but was reduced (60 units in total) when only the parotid glands were injected.

Limitations of this study should be acknowledged. First, due to its retrospective nature, and also the diverse indications for intrasalivary gland botox, the definition of a positive treatment outcome was necessarily broad. Objective tests of sialorrhoea such as sialometry are not appropriate in this cohort and existing scoring systems focus on salivary volume and frequency rather than its effect on quality of life, which are less useful in this context. The Sialorrhea Scoring Scale has been developed specifically for PD, so was not used herein. ${ }^{26}$ For MND, the revised Amyotrophic Lateral Sclerosis Functional Rating Scale includes only one domain, which evaluates sialorrhoea. ${ }^{27}$

The Oral Secretion Scale (OSS) has been designed for the evaluation of sialorrhoea in MND but not for the response to therapy. ${ }^{28}$ The OSS has recently been shown to link to survival, with those with a zero score (severely impaired swallow) dying earlier than those with preserved swallow. Notably, OSS scores of 0 or 1 at initiation of NIV predicted a failure rate of NIV of over $80 \% .^{29}$ Therefore, high symptom burden of sialorrhoea in MND is associated with an inability to comply with long-term home ventilation. There are, however, no data on the minimal clinically important difference for the OSS in terms of treatment effects. Arguably, a global quality of life score may be better placed to capture the treatment benefits of interventions for sialorrhea and should be considered in future trial designs.

Finally, a key limitation is our inability to report any benefit on mortality. Our design was observational and a formal randomised controlled trial would be needed to define this. Although our survival appears to be good, this likely reflects the heterogeneous group included. The ALS/MND cohort reported in a case series had a median survival of 1-11 months dependent on their OSS scores. ${ }^{28}$ In our series, we treated patients with many other conditions. In addition, in our cohort, the decision to try intrasalivary gland botox was taken at varying stages of both sialorrhoea and primary disease severity and would likely be influenced by patient and clinician factors. One factor to consider is that the 'in team' access to this treatment may mean patients had early access to intrasalivary gland botox.

A randomised controlled trial comparing intrasalivary gland botox and standard care as first-line treatments for sialorrhoea using objective outcome measures would further advance the evidence base and provide a case for improving the availability of intrasalivary gland botox to patients living with neurodisability.

\section{CONCLUSION}

This is the largest reported cohort study of intrasalivary gland botox therapy and the first in a home NIV and weaning setting. Our first 109 intrasalivary gland botox treatments were well tolerated and showed benefit across a range of different neurological conditions. Complications were rare. There are clear advantages to patients in having expertise in intrasalivary gland botox injection within a HVS.

Twitter Ben Messer @drbenmesser 
Contributors $\mathrm{JH}$ and BM conceived and designed the study. JH obtained patient data. All authors undertook review and interpretation of the data. JH drafted the initial manuscript. The manuscript was revised, and the final version was approved, by all authors. BM accepts full responsibility for the work and the conduct of the study, had access to the data and controlled the decision to publish.

Funding Merz pharmaceuticals have agreed to pay the open access charges.

Competing interests BM reports speaker fees from Fisher and Paykel outside the submitted work. AdS reports grants and personal fees from AstraZeneca, Bayer, Boehringer, Chiesi, Forest labs, GSK, Grifols, Insmed, Teva, Zambon, outside the submitted work.

Patient and public involvement Patients and/or the public were not involved in the design, or conduct, or reporting, or dissemination plans of this research.

Patient consent for publication Not applicable.

Ethics approval This study does not involve human participants.

Provenance and peer review Not commissioned; externally peer reviewed.

Data availability statement № data are available.

Open access This is an open access article distributed in accordance with the Creative Commons Attribution Non Commercial (CC BY-NC 4.0) license, which permits others to distribute, remix, adapt, build upon this work non-commercially, and license their derivative works on different terms, provided the original work is properly cited, appropriate credit is given, any changes made indicated, and the use is non-commercial. See: http://creativecommons.org/licenses/by-nc/4.0/.

\section{REFERENCES}

1 Hernández LM, Taylor MK. Chapter 2 - Salivary Gland Anatomy and Physiology. In: Granger DA, Taylor MK, eds. Salivary bioscience. Springer, 2020: 11-20.

2 Erasmus CE, van Hulst K, Rotteveel JJ, et al. Clinical practice: swallowing problems in cerebral palsy. Eur J Pediatr 2012;171:409-14.

3 Johnson J, Evatt ML, Chaudhuri KR. Sialorrhoea in Parkinson's disease. In: Ray Chaudhuri K, Tolosa E, Schapira AHV, eds. Nonmotor symptoms of Parkinson's disease. 2nd ed. USA: Oxford University Press, 2014.

4 EFNS Task Force on Diagnosis and Management of Amyotrophic Lateral Sclerosis:, Andersen PM, Abrahams S, et al. EFNS guidelines on the clinical management of amyotrophic lateral sclerosis (MALS)-revised report of an EFNS task force. Eur J Neurol 2012;19:360-75.

5 Hobson EV, McGeachan A, Al-Chalabi A, et al. Management of sialorrhoea in motor neuron disease: a survey of current UK practice. Amyotroph Lateral Scler Frontotemporal Degener 2013;14:521-7.

6 Simonds AK. Progress in respiratory management of bulbar complications of motor neuron disease/amyotrophic lateral sclerosis? Thorax 2017;72:199-201.

7 Parkes J, Hill N, Platt MJ, et al. Oromotor dysfunction and communication impairments in children with cerebral palsy: a register study. Dev Med Child Neurol 2010;52:1113-9.

8 Singh H, Nene Y, Mehta TR, Govindarajan R, et al. Efficacy of botulinum toxin for treating sialorrhea in neuromuscular conditions. Front Neurol 2020;11:513.

9 Kang Y, Chun MH, Lee SJ. Evaluation of salivary aspiration in brain-injured patients with tracheostomy. Ann Rehabil Med 2013;37:96-102.

10 Bourke SC, Tomlinson M, Williams TL, et al. Effects of non-invasive ventilation on survival and quality of life in patients with amyotrophic lateral sclerosis: a randomised controlled trial. Lancet Neurol 2006;5:140-7.
11 Cooksey JA, Sergew A. Noninvasive ventilation in amyotrophic lateral sclerosis. Sleep Med Clin 2020;15:527-38.

12 Vandenberghe N, Vallet A-E, Petitjean T, et al. Absence of airway secretion accumulation predicts tolerance of noninvasive ventilation in subjects with amyotrophic lateral sclerosis. Respir Care 2013;58:1424-32.

13 McGeachan AJ, Hobson EV, Al-Chalabi A, et al. A multicentre evaluation of oropharyngeal secretion management practices in amyotrophic lateral sclerosis. Amyotroph Lateral Scler Frontotemporal Degener 2017;18:1-9.

14 Nice.org.uk, 2013. Available: https://www.nice.org.uk/advice/ esuom15/resources/hypersalivation-oral-glycopyrronium-bromidepdf-54116458942789573 [Accessed 31 Oct 2021].

15 Yu Y-C, Chung C-C, Tu Y-K, et al. Efficacy and safety of botulinum toxin for treating sialorrhea: a systematic review and meta-analysis. Eur J Neurol 2022;29:69-80.

16 Nice.org.uk, 2019. Available: https://www.nice.org.uk/guidance/ ta605/resources/xeomin-botulinum-neurotoxin-type-a-for-treatingchronic-sialorrhoea-pdf-82608898003909 [Accessed 31 Oct 2021].

17 Motor neurone disease: assessment and management. Available: https://www.nice.org.uk/guidance/ng42/chapter/Recommendationsfor-research\#4-saliva [Accessed 18 Jan 2022].

18 Young CA, Ellis C, Johnson J, et al. Treatment for sialorrhea (excessive saliva) in people with motor neuron disease/amyotrophic lateral sclerosis. Cochrane Database Syst Rev 2011;5:CD006981.

19 Jost WH, Friedman A, Michel O, et al. SIAXI. Neurology 2019;92:e1982-91.

20 Hung S-A, Liao C-L, Lin W-P, et al. Botulinum toxin injections for treatment of drooling in children with cerebral palsy: a systematic review and meta-analysis. Children 2021;8:1089.

21 Guidubaldi A, Fasano A, lalongo T, et al. Botulinum toxin A versus $B$ in sialorrhea: a prospective, randomized, double-blind, crossover pilot study in patients with amyotrophic lateral sclerosis or Parkinson's disease. Mov Disord 2011;26:313-9.

22 Møller E, Karlsborg M, Bardow A, et al. Treatment of severe drooling with botulinum toxin in amyotrophic lateral sclerosis and Parkinson's disease: efficacy and possible mechanisms. Acta Odontol Scand 2011;69:151-7.

23 Paracka L, Kollewe K, Klietz M, et al. Incobotulinumtoxina for hypersalivation in patients with amyotrophic lateral sclerosis: an open-label single-centre study. J Neural Transm 2019;126:1341-5.

24 Squires N, Humberstone M, Wills A, et al. The use of botulinum toxin injections to manage drooling in amyotrophic lateral sclerosis/motor neurone disease: a systematic review. Dysphagia 2014;29:500-8.

25 Dogu O, Apaydin D, Sevim S, et al. Ultrasound-guided versus 'blind' intraparotid injections of botulinum toxin-A for the treatment of sialorrhoea in patients with Parkinson's disease. Clin Neurol Neurosurg 2004;106:93-6.

26 Abdelnour-Mallet M, Tezenas Du Montcel S, Cazzolli PA, et al. Validation of robust tools to measure sialorrhea in amyotrophic lateral sclerosis: a study in a large French cohort. Amyotroph Lateral Scler Frontotemporal Degener 2013;14:302-7.

27 Cedarbaum JM, Stambler N, Malta E, et al. The ALSFRS-R: a revised ALS functional rating scale that incorporates assessments of respiratory function. BDNF ALS Study Group (phase III). J Neurol Sci 1999;169:13-21.

28 Brooks BR, Lewarski JL, et al. Oral secretion scale (OSS) score in amyotrophic lateral sclerosis patients is associated with tolerance of non-invasive positive pressure ventilation, need for hospice or transition to tracheal positive pressure ventilation (TPPV) and survival (Abstract). Amyotroph Lateral Scler 2010;11:140.

29 Cazzolli PA, Brooks BR, Nakayama Y, et al. The oral secretion scale and prognostic factors for survival in subjects with amyotrophic lateral sclerosis. Respir Care 2020;65:1063-76. 